\title{
Voluntariedad, género e interés por la política en el asociacionismo de Japón. Un estudio de caso
}

\author{
Voluntariness, gender and interest in politics \\ in Japanese associationism. A case study
}

\author{
KYOKO ITO-MORALES \\ Universidad de Granada \\ RAFAEL VÁZQUEZ-GARCÍA \\ Universidad de Granada
}

\section{Cómo citar/Citation}

Ito-Morales, K. y Vázquez-García, R. (2017). Voluntariedad, género e interés por la política en el asociacionismo de Japón. Un estudio de caso. Revista Española de Ciencia Política, 44, 181-207. Doi: https://doi.org/10.21 308/recp.44.07

\section{Resumen}

Los estudios sobre la participación en asociaciones cívicas voluntarias muestran una relación positiva con la participación política. Este trabajo estudia si el tipo de participación (voluntaria o no) se relaciona de la misma manera con el nivel de interés por los asuntos sociopolíticos, usando para ello evidencias del caso de Japón. Los resultados apuntan que la distinción entre participación voluntaria y no voluntaria resulta especialmente relevante para el caso de los hombres. Asimismo, los resultados indican que las variables de recursos del modelo de voluntarismo cívico parecen ser las más relacionadas con el interés por la política, excepto en el caso de las mujeres, donde no se sigue la misma pauta.

Palabras clave: participación cívica, asociaciones voluntarias, voluntarismo cívico, diferencias de género, interés político, Japón.

\begin{abstract}
Studies on participation in voluntary civic associations show a positive relationship with political participation. This work examines whether the type of participation (voluntary or not) is related to the level of interest in socio-political issues in the same way. To that aim, it uses evidences from the case of Japan. The results suggest that the distinction between voluntary and non-voluntary participation is especially relevant for men. Likewise, the results indicate that
\end{abstract}


the resource variables of the civic voluntarism model seem to be the most strongly related to the interest in politics; but the same pattern is not followed in the case of women.

Keywords: civic participation, voluntary associations, civic voluntarism, gender gap, political interest, Japan.

\section{INTRODUCCIÓN}

La potencial relación positiva entre la participación en asociaciones cívicas y sus efectos beneficiosos sobre el funcionamiento de la democracia ha sido largamente estudiada (Tocqueville, 2003; Putnam et al., 1993; Putnam, 2000; Warren, 2001; Newton, 1999; Etzioni, 1993; Etzioni et al., 1994; Verba et al., 1995; 2002; Boix y Posner, 1998; Cohen y Rogers, 1992, entre otros). Desde el célebre libro La democracia en América de Tocqueville (2003, del original de 1835-1840) sobre las asociaciones y su papel democratizador en la vida de los Estados Unidos de América, a los trabajos de Almond y Verba (1963), o los más recientes de Putnam et al., (1993) y Putnam (2000), con su famosa tesis sobre el capital social, las asociaciones voluntarias han sido percibidas de forma positiva por su papel como «escuelas de democracia». Sin embargo, el grado de «voluntariedad» o «no voluntariedad» en la involucración en estas asociaciones se ha venido ignorando, con pocas excepciones como las de Ellison y Sherkat (1995), o de Ayala (2000). No obstante, en este trabajo sostenemos que la voluntariedad o no de los miembros en las asociaciones cívicas afecta al aprendizaje de las habilidades cívicas y al interés en política. Para testar esta hipótesis, se analiza la relación empírica entre voluntariedad o no voluntariedad de la participación en las asociaciones cívicas voluntarias y el interés en política con datos de encuesta. Los resultados del análisis muestran una clara relación entre dichas variables. Por otra parte, si bien se reseñan diferencias en relación con el género, estas no resultan tan explícitas ni tan contundentes. El trabajo pretende subrayar la necesidad de seguir indagando en los efectos negativos hacia un interés por los asuntos públicos que puede tener la falta de voluntariedad real y efectiva a la que se enfrentan los ciudadanos de algunos sistemas políticos como el caso de Japón, al tiempo que alentar una mayor profundización en torno a las diferencias de género para explicar el recurrente excepcionalismo femenino que suele observarse en muchas sociedades y no solo en la japonesa.

Con este objetivo se comienza con una reflexión teórica en torno a la relación entre voluntariedad participativa en el seno de las asociaciones y los efectos sobre el interés por la política. Seguidamente se justifica la singularidad de Japón como objeto de estudio y se presentan sus particularidades históricas, sociales y políticas a la hora a abordar el estudio de la vida cívica y el asociacionismo. En la segunda parte del artículo, tras detallar tanto el modelo explicativo como el diseño y la metodología de la investigación, nos centraremos en el análisis y la discusión de los resultados. 


\section{VOLUNTARIEDAD, NO VOLUNTARIEDAD Y EL INTERÉS EN POLÍTICA: CONSIDERACIONES TEÓRICAS}

El centro de interés de este trabajo es si la voluntariedad o no voluntariedad para participar en unas asociaciones cívicas afecta a los miembros de las asociaciones en relación con su interés político. Desde una posición republicana cívica, entendemos que el correcto desarrollo de las democracias depende en buena medida de la implicación política de los ciudadanos, y para que estos estén involucrados en los asuntos públicos hace falta un cierto interés por los temas sociopolíticos; es decir, el interés por la política debiera constituir un elemento consustancial a la democracia (Barber, 1984; Bellah et al., 1987).

Existen diversas modalidades de mostrar y ejercer el interés por la política: votar, donar dinero a los partidos políticos, asistir a las campañas o reuniones políticas, etc. No obstante, hay otras maneras más indirectas de acercarse a la vida política. Las teorías de la sociedad civil, el capital social y el voluntarismo cívico plantean el potencial de las asociaciones voluntarias como mecanismo para encauzar el interés político y la participación, puesto que ellas podrían promocionar en sus miembros el interés y la capacidad para poder vincular sus preocupaciones cercanas con los problemas más lejanos (abstractos), confiar en otros miembros para trabajar juntos y adquirir habilidades cívicas como organizar reuniones, hacer presentaciones públicas o escribir cartas a los políticos, entre otras posibilidades (Persell et al., 2001; Putnam et al., 1993; Putnam, 2000; Verba et al., 1995).

La práctica totalidad de los estudios que muestran este tipo de efectos positivos de las asociaciones cívicas voluntarias parecen presuponer que los participantes o voluntarios están involucrados en ellas siempre de forma voluntaria. La libertad de asociación y participación es un derecho fundamental en la democracia liberal, y aun los defensores de la sociedad civil para la mejora de la democracia basan, por lo general, sus pensamientos en la tradición liberal. Por lo tanto, la cuestión de voluntariedad y de no voluntariedad se considera como una premisa resuelta de antemano. No obstante, como argumenta Bell (2016), existen factores culturales, mayoritariamente no occidentales, que afectan a la prioridad y posibilidad real de libertad, planteando que a menudo los miembros de esas culturas aceptan la participación en asociaciones cívicas por obligación más que por su convencimiento (ibid.:150).

Entendemos la sociedad civil como el área de actividades sociales que incluye las instituciones y asociaciones cívicas que generalmente se consideran asociaciones voluntarias tales como organizaciones de calidad, organizaciones sin ánimo de lucro, movimientos sociales, organizaciones religiosas, asociaciones de vecinos, clubes de deportes, asociaciones de profesores y padres y madres, asociaciones profesionales, grupos de consumidores, etc., y a menudo se refieren a los participantes en ellas como voluntarios. Se trata de un espacio de asociaciones que actúan en la sociedad, pero que se sitúan en algún lugar intermedio entre el individuo y su esfera privada y las relaciones públicas y mercantiles (Vázquez, 2010: 44). 
El reconocimiento más reciente de la sociedad civil y las asociaciones cívicas se origina a partir de los debates entre la universalidad del liberalismo argumentada por Rawls (1971), y las réplicas de los filósofos políticos republicanos como Sandel (1998), además de la situación política sociológica del final de los años ochenta, con la caída del bloque comunista, que abrió las oportunidades de participación en política en estas zonas (Vázquez, 2010: 78).

Otros autores ponen el énfasis en la responsabilidad social como forma de reparar una sociedad fragmentada por la falta de virtudes cívicas (Etzioni, 1993; Bell, 2016). Persell y otros subrayan el papel regulador ético y moral de las asociaciones de la sociedad civil y el rol educativo de sus miembros para que desarrollen lealtad y confianza entre ellos, enseñen prácticas sociales como urbanidad y cooperación, y cuiden la seguridad de sus miembros y de la sociedad (Persell et al., 2001: 206). Las habilidades positivas que se esperan adquirir a través de esas asociaciones son, entre otras: trabajar juntos, cooperar unos con otros para lograr metas comunes, pensar más allá de sí mismos, cuidar de los asuntos más lejanos de sus comunidades cercanas o interesarse en los asuntos sociopolíticos. Es por ello que decimos que la sociedad civil es escuela de virtudes (Rosenblum, 1998: 26) y las asociaciones cívicas voluntarias son escuelas de democracia (Morales, 2002: 498).

El concepto y las definiciones de capital social son variados y eso provoca cierta confusión y pérdida de poder explicativo en la teoría (Maan, 2011: 10). En este trabajo asumimos la versión ofrecida por Putnam et al., (1993), y Putnam (2000) y el argumento consistente en defender la relación positiva entre participación en asociaciones voluntarias y la mejora de la calidad de la democracia. Según su definición, el capital social hace referencia a las conexiones entre los individuos y las redes sociales, así como de las normas de reciprocidad y de confianza que nacen de ellas (Putnam, 2000: 19). La idea consiste en que para que se desarrollen los hábitos y capacidades mencionados, los partidarios de las virtudes del capital social crean que las asociaciones cívicas voluntarias poseen precisamente esta potencialidad para educar a sus miembros cívicamente, estimular que ellos relacionen sus intereses privados con los asuntos comunes o sociales y, finalmente, tengan capacidad para promocionar una participación activa en la política de su país y su democracia (Cohen y Rogers, 1992; Pateman, 1970; Putnam et al., 1993; Putnam, 2000; Warren, 2001; Newton, 1999; Etzioni, 1993; Etzioni et al., 1994; Verba et al., 1995; Verba et al., 2002; Boix y Posner, 1998).

Wollebaek y Selle $(2002 ; 2003)$ dudan, no obstante, de la perentoria necesidad de capital social generado entre los miembros de asociaciones voluntarias a través de los contactos "cara a cara» en los que Putnam pone un énfasis e importancia especial, y demuestran que la participación en estas asociaciones mediante una participación pasiva representada por la contribución económica también mejora el capital social y la participación en la política.

Por último, se presenta el modelo de voluntarismo cívico o también llamado modelo socio-económico-estado (modelo-SES) de Verba et al. (1995). Según ellos, la participación política depende no solo del involucramiento de los ciudadanos en las asociaciones e instituciones políticas sino también en instituciones sociales como la 
familia, escuelas, lugares de trabajo, asociaciones voluntarias e instituciones religiosas (iglesias), puesto que también estos ámbitos ofrecen posibilidades de adquirir habilidades cívicas, circunstancias y decisiones de vida que marcan formas y maneras de participar en las actividades políticas. Para ellos, la adquisición de las habilidades cívicas es muy importante porque esas habilidades comunicativas y de organización, como planear y organizar reuniones, escribir cartas y hacer presentaciones o discursos, son transformables en los ámbitos políticos y disponen a los ciudadanos a utilizar su tiempo y dinero eficazmente en la vida política, lo que animaría a que estén interesados en política y participen en ella (Verba et al., 1995: 304).

La pregunta principal que plantean todos estos modelos explicativos es: «iPor qué algunas personas participan en la política y otras no?». Y las razones posibles para los no participantes pueden ser "porque no pueden", "porque no quieren», o "porque nadie pregunta» (Verba et al., 1995: 15). Así, estos autores se concentran en analizar las variables de recursos (conocimiento y habilidades, dinero y tiempo), las redes de reclutamiento y la motivación (o la orientación sobre la política), y concluyen que los mismos están relacionados con la participación política (Burns et al., 2001: 33, 365; Schlozman et al., 2012: 18-21). El modelo de voluntarismo cívico es interesante para el presente estudio porque se menciona singularmente la importancia de la motivación: para que los ciudadanos estén activos en política necesitan tener un cierto nivel de motivación y deseo, es decir, la voluntariedad para participar en ella (Verba et al., 1995: 391). Sin embargo, la evidencia proveniente de encuestas no es completamente concluyente, puesto que la mayor parte de los estudios no disponen de suficientes casos como para analizar la relación entre involucrarse en asociaciones sociales y el interés en la política (ibid., 1995: 392).

De este modo, surge la pregunta acerca de si la voluntariedad de participación en las asociaciones voluntarias no requiere estudios más profundos que cuestionen más ampliamente la suposición de que todos los participantes en las asociaciones voluntarias tienen el mismo grado de voluntariedad. Esto es, cabría preguntarse si todas las personas involucradas de una u otra forma en asociaciones voluntarias lo hacen desde la plena voluntariedad democrática como libre elección o, por el contrario, se encuentran impelidas a hacerlo por distintos condicionantes sociales, familiares, culturales, etc. Desde el estudio de Tocqueville (2003) sobre la sociedad americana, muchos de los investigadores sobre el capital social han dado por hecho que las asociaciones benéficas para la mejora de la democracia son siempre de carácter voluntario. La común asunción es que el sector voluntario es el protagonista de la sociedad civil, y en él no deben incluirse las relaciones familiares, el mercado ni el Estado, y además, se presupone no influido por estas esferas (Newton, 1999: 10). Los miembros de las asociaciones voluntarias se unirían por sus libres decisiones, puesto que hacer trabajo voluntario significa elegir su acción sin espera de compensación monetaria, aunque vaya más allá de sus obligaciones básicas (Ellis y Campbell, 2005: 4; Burns et al., 2001: 4); por lo tanto, los voluntarios no deben estar obligados por las autoridades o las culturas. Además, podría añadirse que los voluntarios estarían igualmente conectados por una estructura horizontal que asegurase la igualdad entre los miembros (Verba et al., 2002). 
Sin embargo, la voluntariedad de los miembros de las asociaciones no parece que esté garantizada siempre de forma completa. Podría plantearse la existencia de miembros que están participando en sus actividades no porque quieran sino porque no pueden evitarlo. En algunos de sus trabajos, Dekker y Halman han identificado tres razones por las que la gente participa en asociaciones voluntarias: a) las personas especialmente involucradas en el activismo con los niños suelen hacer trabajos voluntarios para niños porque ellos mismos demandan similares tipos de servicios; b) la gente que vive en las zonas rurales participa a menudo en actividades voluntarias, puesto que los servicios necesitados generalmente ofrecidos por las autoridades locales no llegan hasta esos lugares, por lo que no hay más remedio que cooperar unos con otros para cubrir la falta de servicio, y c) hay también personas que simplemente quieren trabajar voluntariamente por su deseo de trabajar para el bien común (Dekker y Halman, 2003: 6). Sin embargo, algunos autores como Wollebaek y Selle argumentan que generalmente los motivos personales y «egoístas» son más frecuentes como causas de participación en asociaciones voluntarias mientras que las razones más «altruistas» serían mucho menos comunes (2003: 78).

En realidad, hay gente que hace trabajos dentro de las asociaciones voluntarias, pero no necesariamente por su voluntad, sino más bien por necesidad. Es decir, la motivación o la voluntariedad altruista para la participación en las asociaciones voluntarias tal como explican Ellis y Campbell (2005) o Burns (2001) no es siempre afirmativa. Resulta muy complicado en ocasiones determinar cuándo personas participan en actividades cívicas sociales bajo su libre voluntad y cuándo sin voluntad. Igualmente es posible que cambie la voluntariedad de pertenencia en las asociaciones voluntarias a no voluntariedad a lo largo del tiempo. Por ejemplo, alguien que ya es miembro de una asociación voluntaria y participa en ella de forma voluntaria, pero que debido a algunos cambios vinculados a situaciones sociodemográficas como la edad, el nivel de ingresos, el nivel de educación, etc., o bien a situaciones de relaciones humanas dentro de la asociación, experimenta el paso de una voluntariedad a una no voluntariedad. Los participantes pasivos eligen un tipo de participación de este tipo para defender su autonomía individual, pero algunos cambios de situación personal, motivación y recursos disponibles, etc., puede que conviertan a los participantes pasivos en activos (Wollebaek y Selle, 2003: 82). Burns et al. (2001: 5) también reconocen que la diferenciación entre participación voluntaria y el empleo pagado es muy borrosa. Otros factores tales como la educación recibida, las orientaciones familiares, las experiencias previas de trabajos voluntarios o la religiosidad, también afectan a la voluntariedad o no voluntariedad. Verba et al. (1997) y Burns et al. (2001) se interesan especialmente en la diferencia de género sobre la motivación o voluntariedad en el asociacionismo. Podríamos afirmar que desde el punto de vista teórico-filosófico de la tradición liberal, es muy difícil y complejo justificar la «voluntariedad» o «no voluntariedad» asociativa. Tal vez por ello, podríamos suponer que la ausencia de debates sobre la voluntariedad y no voluntariedad en los estudios previos se debe a la dificultad para justificar la voluntariedad teóricamente.

Aun así, si buscamos en la literatura fuera del ámbito liberal, concretamente en el Este Asiático, existen algunas interpretaciones alternativas. Bell $(2006 ; 2016)$ destaca la prioridad de las obligaciones sociales y familiares sobre los derechos de participación 
política en los países con influencia del confucianismo. Beng-Huat (1999: 573) admite que la tradición confuciana justifica el poder autoritario sobre los individuos para que se sientan obligados a formar parte de un grupo o una comunidad con el propósito de mantener la armonía social. Además, Chan (1997: 40) añade que la mayoría de los asiáticos del este se identifican con tal estilo de participación. La falta de desarrollo de sociedad civil sostenida por las asociaciones voluntarias, tal como se define en la teoría liberal occidental, estaría provocada por ese contexto cultural diferente (Tu, 1996: 8). En esta cultura, la participación en las asociaciones cívicas es frecuentemente obligatoria, aunque esas asociaciones se categoricen dentro de las asociaciones voluntarias en las encuestas sociales convencionales.

Esta investigación no pretende profundizar en el debate filosófico-teórico acerca de la voluntariedad y no voluntariedad, aunque se reconoce de antemano la dificultad de una justificación teórica partiendo del liberalismo. Tampoco se pretende extender los debates sobre la universalidad de las teorías occidentales en otras sociedades como el mundo asiático, sino concentrarse en el análisis de la influencia de la voluntariedad o no voluntariedad de los participantes de asociaciones cívicas sobre el interés por la política, y su posible efecto distinto entre hombres y mujeres.

Existen algunos estudios que han tratado el factor de voluntariedad y no voluntariedad y las asociaciones cívicas. Ellison y Sherkat (1995) buscan razones y condiciones socioculturales que favorecen la existencia de las instituciones no voluntarias. Sus enfoques se concentran en las iglesias americanas de los afroamericanos, y los resultados muestran que las iglesias afroamericanas en las zonas rurales del sur se caracterizan por ser «semi-no-voluntarias», es decir, la participación en las actividades de la congregación está fuertemente motivada por las normas sociales o la presión social, por tener los aspectos de multifuncionalidad y centralidad simbólica, además de por la ausencia de recursos seculares para posiciones sociales, asistencia social y la falta de liderazgo, mientras que la participación en las iglesias afroamericanas en las zonas urbanas es más voluntaria (Ellison y Sherkat, 1995: 1416-1419). Aunque su investigación se centra en las iglesias afroamericanas, las características, condiciones y ambientes mencionados de las instituciones semi-no-voluntarias son compartidas con otras asociaciones generalmente categorizadas como voluntarias tales como asociaciones de vecinos, asociaciones de profesores y padres y madres y asociaciones profesionales en algunos países o algunas zonas, con lo cual sería interesante extender su estudio a otras asociaciones cívicas.

Otra investigación llevada a cabo por Ayala trata la diferencia entre los miembros de asociaciones voluntarias e involuntarias sobre la participación en política. Cuestionando los resultados de los estudios de Verba et al. (1995) sobre las habilidades cívicas, Ayala estudia la diferencia entre asociaciones voluntarias y los grupos formados en el lugar de trabajo concluyendo que los miembros de asociaciones voluntarias muestran más participación activa en la política que los de los grupos involuntarios (Ayala, 2000). Los grupos formados voluntariamente, pero que se sitúan en el lugar de trabajo tienden a ser menos voluntarios y los miembros actúan con menor voluntariedad debido a la naturaleza laboral de las relaciones, puesto que cualquier decisión que se tome podría estar sometida a vigilancia y escrutinio y, por ende, significar un riesgo en 
su estabilidad laboral. Por lo tanto, las habilidades que uno puede aprender en los grupos voluntarios relacionados con el lugar de trabajo nunca van a ser las mismas que se pueden adquirir en las asociaciones voluntarias que se sitúen al margen de las relaciones laborales. De igual modo, en las asociaciones voluntarias exógenas al trabajo se podrían desarrollar más habilidades cívicas que en el seno de los grupos relacionados con el lugar laboral, donde se tendería a promocionar más las habilidades vinculadas y necesarias para esas actividades laborales (Ayala, 2000: 101). Se concluye, por tanto, que debería tenerse en cuenta que la motivación (cursiva en el texto original) que se encuentra detrás de la participación en diferentes asociaciones, mediatiza la relación entre participación en asociaciones y la participación política (Ayala, 2000: 109).

Por último, Tiefenbach y Holdgrün (2015) analizan dos bases de datos diferentes sobre la sociedad japonesa y descubren que la voluntariedad para participar en las asociaciones de vecinos está correlacionada con mayores niveles de implicación social y compromiso cívico. Este estudio muestra que la participación política y la participación en la comunidad a través de las asociaciones voluntarias generan capital social y altos niveles de felicidad entre sus miembros. De este modo, enfocan su análisis en las asociaciones de vecinos en Japón porque les ofrecen posibilidades de analizar los tres aspectos mencionados (Tiefenbach y Holdgrün, 2015: 73-77). Además, muestran que las diferencias de género tienen influencia en la voluntariedad y no voluntariedad de participación en las asociaciones cívicas y la felicidad. En este sentido, una ya abundante literatura ha mostrado previamente que las mujeres parecen estar menos interesadas en la política que los hombres (Pérez-Fuentes, 1990; Delli Carpini y Keeter, 1992; Inglehart y Norris, 2003; Wilson, 2007; Verge y Tormos, 2012; Fraile y Gómez, 2017). Algunos factores podrían ayudar a explicar ese hallazgo: la falta de tiempo por cuidar a los niños además de trabajar, el cuidado de la familia que absorbe la capacidad física y mental para preocuparse de la política, la estructura tradicional patriarcal de familia que discrimina las funciones de las mujeres, la falta de recursos socioeconómicos o el proceso de socialización que hace que las mujeres tengan menos oportunidades en el ámbito político (Burns et al., 2001: 7-8).

Parece quedar claro después de todo lo anterior que, aunque los presupuestos liberales en los que se asienta la mayor parte de las teorías del capital social y de la participación asociativa asumen de facto la voluntariedad consciente y la libertad jurídica de los individuos para vincularse a los procesos participativos, no es desdeñable el número de factores que potencialmente podrían distorsionar el carácter voluntario de este compromiso. Circunstancias sociales, históricas, económicas, comunitarias o relacionadas con el género cuestionan no pocas veces la aceptada voluntariedad plena de las decisiones para ser partícipes en los mecanismos asociativos.

\section{JAPÓN COMO OBJETO DE ESTUDIO Y SUS PARTICULARIDADES}

Para examinar el efecto de la voluntariedad o no voluntariedad en las asociaciones cívicas sobre el interés por la política hemos elegido el caso de Japón. La sociedad 
japonesa parece contener un importante número de asociaciones y grupos cívicos voluntarios con falta de voluntariedad, tal y como ocurriría en el caso ejemplificado de las asociaciones de vecinos (Yamazaki, 1999; Torigoe, 1994; Tanaka, 2000).

La mayoría de los académicos están de acuerdo en que no se puede comparar la sociedad civil japonesa con las del mundo occidental, puesto que son distintas (Schwartz y Pharr, 2003; Pekkanen, 2004, 2006; Kingston, 2004; Vinken, et al., 2010). La imagen de sociedad civil japonesa que se puede encontrar en la bibliografía más reciente puede dividirse en dos tipos: por un lado, la sociedad civil en Japón es poco activa, sin asociaciones secundarias relevantes; por otro, hay que quienes defienden la existencia de un buen número de asociaciones y grupos locales activos y con vida. Esa confusa imagen está causada por unas características particulares de las asociaciones cívicas en Japón. Por ejemplo, muchas asociaciones pequeñas, especialmente los grupos locales, tienen un rasgo informal (Pekkanen, 2006), y otras, aun siendo más formales y grandes, mantienen una relación de interdependencia con las autoridades o gobiernos locales (Schwartz y Pharr, 2003; Haddad, 2007, 2010). Además, numerosas asociaciones se sostienen no por la unidad voluntaria de sus miembros, sino más bien por la presión social (Pekkanen, 2004; 2006).

Las asociaciones de vecinos, entre otras, son las más representativas de las asociaciones cívicas con las características típicas japonesas: son más de 300000 y se encuentran a lo largo de toda la geografía japonesa (Pekkanen, 2006). Supuestamente, son asociaciones voluntarias independientes y autónomas para favorecer el autogobierno de barrio, pero a la vez están promocionadas por las autoridades locales y el Gobierno central para poder transferir información a barrios vecinos con efectividad o reducir el coste de los servicios sociales. A todo ello se suma que la presión social o la cultura tradicional colectivista obliga de alguna manera a la mayoría de personas en Japón a que formen parte de ellas (Yamazaki, 1999: 30-31; Torigoe, 1994: 9). Por otro lado, a pesar de la existencia de numerosas asociaciones voluntarias, a los japoneses parece que no les interesa participar en asuntos políticos, aunque muchas asociaciones cívicas con profunda relación de interdependencia con los gobiernos (incluyendo las asociaciones de vecinos) estén estrechamente relacionadas con temas políticos. Todo ello sugiere que los japoneses prefieren que los problemas políticos estén en manos de los políticos profesionales (Vinken et al., 2010: 7).

De otro lado, las mujeres tienen muy poca presencia en la política japonesa. Si hablamos de representación política, según el informe de la Unión Interparlamentaria de $2015^{1}$, el porcentaje de las mujeres en la Cámara Baja en Japón es solo del 9,5\% y en la Cámara Alta del 15,7\%. En el ranking total de representación parlamentaria femenina, Japón ocupa el puesto 114 de 140 países, muy por debajo de la media y el peor de los países desarrollados. Algunos estudios muestran asimismo que el nivel de participación en actividades sociopolíticas no electorales por parte de las mujeres es

1. Disponible en: http://www.ipu.org/wmn-e/classif.htm. 
bajo (Takeda, 2010; Masuyama y Yamada, 2008; Ōyama, 2002). Pekkanen (2006: 183) muestra a su vez que las organizaciones de mujeres en Japón son especialmente débiles en cuanto a presencia. Gelb (2003) hace hincapié en el sistema político institucional, que desfavorece la participación política de las mujeres japonesas, mientras Pye y Pye (1985) argumentan que la influencia cultural, sobre todo el confucianismo, divide los roles sociales por género y las mujeres no están designadas para la política. LeBlanc (1999: 69) observa una presión social particularmente importante en las madres para mantenerse fuera de la arena política. Sin embargo, en la actualidad las mujeres japonesas van cambiando sus comportamientos políticos progresivamente. Así, un reciente estudio muestra que las mujeres están participando más activamente en la política, especialmente en asuntos relacionados con sanidad, seguridad de alimentos y cuidado de niños, si bien se trata de una tendencia relativamente nueva, surgida después del gran terremoto de Japón Oriental en 2011 y la contaminación radioactiva causada por él (Holdgrün y Holthus, 2014).

En resumen, las teorías de la sociedad civil, el capital social y los modelos occidentales de voluntarismo cívico deben enfrentarse a un importante desafío a la hora de analizar las incidencia del voluntarismo cívico en Japón y su incidencia en el interés por la política. Y ello por cuatro razones: a) la falta de voluntariedad en algunas asociaciones voluntarias; b) la falta de interés en la política en general; c) la falta de voluntariedad en la participación cívica, que se correlaciona con el bajo interés por la política, y, por último, d) las diferencias de género que parecen afectar igualmente al nivel de interés en la política además del efecto de la voluntariedad o no.

\section{MODELO EXPLICATIVO, DISEÑO Y METODOLOGÍA}

A partir de las anteriores consideraciones y teniendo en cuenta los estudios mencionados en la sección anterior, las asociaciones cívicas japonesas con sus particularidades constituye una oportunidad para estudiar unos temas que requieren, en cualquier caso, de investigaciones más a fondo. Así, se ha diseñado esta investigación con los siguientes objetivos:

1. Estudiar la relación entre participación en las asociaciones cívicas y el interés en la política en el caso de Japón.

2. Comprobar si la voluntariedad o no voluntariedad de participar en las asociaciones cívicas afecta al interés en política en Japón.

3. Identificar si existen diferencias de género relevantes

En cuanto a las hipótesis, la primera sigue las teorías de sociedad civil y capital social y voluntarismo cívico, es decir, la existencia de una correlación positiva entre la participación en las asociaciones cívicas y el interés en política. Por lo tanto: 
HO. Las personas que participan en las actividades cívicas tienen más interés en la politica en Japón.

Segundo, a partir de esta hipótesis, y asumiendo que las asociaciones cívicas japonesas se caracterizan por la falta de voluntariedad de participación, se puede afirmar que los motivos de los participantes en las asociaciones voluntarias en Japón no siempre coinciden con su libre voluntad. En consecuencia, las motivaciones de involucrarse en actividades voluntarias o relacionarse con los miembros de las mismas, las actitudes e intereses de contribuir en las actividades y, finalmente, la facilidad de ampliar sus intereses sociales a problemas sociales más grandes -a saber, interesarse en los asuntos sociopolíticos-, pueden variar dependiendo de la voluntariedad o la no voluntariedad para participar en las asociaciones cívicas. Así pues, se plantea la siguiente hipótesis:

H1. La participación en asociaciones aumenta siempre el interés por la política, tanto en el caso de la participación voluntaria como en el de la no voluntaria.

Al mismo tiempo, como hemos presentado a través de los estudios de Burns et al. (2001); Tiefenbach y Holdgrün (2015); Takeda (2010); Masuyama y Yamada (2004), y Ōyama (2002), la relación entre la voluntariedad de la participación en las asociaciones secundarias y el interés por la política será distinta para hombres y mujeres, de lo que se deduce la hipótesis siguiente:

H2. El efecto de la voluntariedad de la participación en asociaciones sobre el interés por la politica es de distinta magnitud para hombres y mujeres.

Para dar respuesta a los objetivos planteados, utilizamos la base de datos National Survey of Lifestyle Preferences (Encuesta Nacional sobre las Preferencias de Estilos de Vida -ENPEV-, kokumin seikatsu senkō do chōsa) ${ }^{2}$. Desde 1995, la Oficina de Gabinete del Gobierno japonés realiza las encuestas ENPEV. Los enfoques de las encuestas varían de un año a otro considerando los intereses de los ciudadanos y el ambiente social.

La presente investigación se basa en la encuesta de ENPEV realizada en marzo de 2012 y publicada en junio de 2013, la más reciente de la serie disponible. Además, es una de las pocas encuestas que contiene variables que preguntan directamente sobre la voluntariedad o no voluntariedad de participar en las actividades cívicas. La encuesta de 2013 tiene su enfoque en la percepción y la opinión declarada de los ciudadanos sobre las actividades cívicas y las asociaciones voluntarias que la Oficina de Gabinete japonesa denomina «el nuevo público común (Atarashii kōkyō)». En el procedimiento aleatorio en dos etapas y estratificado, unos 4000 hombres y mujeres japoneses de edades entre quince y ochenta años fueron elegidos para participar en esta encuesta. Está

2. El resumen de las encuestas realizadas se puede consultar visitando la página siguiente (disponible sólo en japonés): http://www5.cao.go.jp/seikatsu/senkoudo/senkoudo.html. 
realizada por una agencia delegada por la Oficina de Gabinete, y los examinadores de la misma empresa visitaron a los participantes para explicar la encuesta y recoger las respuestas (modalidad de administración cara a cara). Finalmente, unas 2802 personas completaron los cuestionarios y la tasa de respuesta fue del $70,1 \%$.

Para conocer el interés por la política, que es la variable dependiente de nuestra investigación, se utiliza la pregunta sobre el grado de acuerdo o desacuerdo con la afirmación: «Yo tengo interés en los problemas sociales y los asuntos políticos más que otras personas", ofreciéndose a los encuestados una escala con cuatro posibles respuestas: 1. Totalmente de acuerdo. 2. Más o menos de acuerdo. 3. Más o menos en desacuerdo. 4. Totalmente en desacuerdo. La utilizamos como variable numérica para la estimación.

Como variables independientes hemos utilizados varias. Así, para saber la densidad de la participación en las asociaciones cívicas, se crea una variable que mide la suma de las actividades cívicas de participación preguntadas en el cuestionario (actividades de educación, cuidar a los niños, desarrollar la comunidad, prevención de crímenes y desastres, cuidar a los mayores y bienestar, actividades académicas y deportes, medioambiente, rescate y recuperación de desastres, cooperación internacional, actividades relacionadas con la reconstrucción del gran terremoto del este de Japón, promoción de las TIC, desarrollo de la habilidad de trabajo, protección del interés de los consumidores y otras). Estas modalidades participativas incluyen formas de participación directa en ellas, participación por utilizar los servicios de ellas, y la participación mediante donación.

Además, construimos una serie de variables dicotómicas para medir la voluntariedad e involuntariedad de participar en las actividades mencionadas. Los criterios utilizados para dividir entre voluntariedad y no voluntariedad son acordes con la definición de los miembros de asociaciones voluntarias presentada anteriormente (Ellis y Campbell, 2005: 4 y Burns et al., 2001: 4). Así, si los encuestados eligen alguna de las siguientes razones para participar en las actividades cívicas: «Porque siento una motivación en mi vida», "porque quiero contribuir a la sociedad», "porque disfruto participando en ellas», o "porque puedo hacer amistades», se les da un valor de 1 como variable de voluntariedad de participación. En cambio, si contestan «porque tienen que ayudarse mutuamente», "porque quiero aumentar ingresos»o "porque es obligatorio participar puesto que los cargos turnan", se les da un valor de 0 como variable de no voluntariedad de participación. De esta forma, la conversión de la variable en numérica nos permite aplicar los supuestos de la regresión lineal.

En cuanto a las variables de control, se incluyen las variables socio-demográficas que constituyen los habituales factores que tener en cuenta a la hora de predecir el interés por la política (edad, sexo, ingreso anual de la familia ${ }^{3}$, estado civil, el número de hijos, la profesión laboral, y los tipos de contrato laboral). Estimamos el efecto de la voluntariedad en el interés por la política a través de una ecuación de regresión lineal.

3. El ingreso anual de la familia categoriza con: $1=$ menos de 2000000 yenes; $2=$ menos de 4000000 yenes; 3 = menos de 6000000 yenes; $4=$ menos de 8000000 yenes; $5=$ menos de 10000000 yenes; 6 = menos de 12000000 yenes; $7=14000000$ yenes, y $8=$ más de 14000000 yenes. 


\section{ANÁLISIS Y DISCUSIÓN DE LOS RESULTADOS}

La tabla 1 muestra que cuanta más participación cívica, mayor interés por la política. No obstante, la magnitud del efecto es pequeña (0.072). De igual forma, a mayor número de hijos, menor interés en política. Estos resultados corroboran el modelo SES de voluntarismo cívico. Así, los que tienen más recursos económicos (mayores

TABLA 1.

REGRESIÓN LINEAL DE INTERÉS EN POLÍTICA (VS. PARTICIPACIÓN EN ACTIVIDADES CÍVICAS)

\begin{tabular}{|c|c|c|}
\hline \multicolumn{3}{|c|}{ (Variable independiente) } \\
\hline Participación en actividades cívicas & $\begin{array}{c}0.072 \\
(0.004)\end{array}$ & $* * *$ \\
\hline \multicolumn{3}{|c|}{ (Variables de control) } \\
\hline Sexo & $\begin{array}{c}0.14 \\
(0.034) \\
\end{array}$ & $* * *$ \\
\hline Edad & $\begin{array}{c}0.278 \\
(0.001)\end{array}$ & $* * *$ \\
\hline Ingresos de la familia & $\begin{array}{l}0.105 \\
(0.01)\end{array}$ & $* * *$ \\
\hline Casado/a & $\begin{array}{l}-0.008 \\
(0.056)\end{array}$ & \\
\hline Número de hijos/as & $\begin{array}{c}-0.074 \\
(0.019)\end{array}$ & ** \\
\hline Hijos/as menores de 6 & $\begin{array}{c}0.04 \\
(0.06) \\
\end{array}$ & \\
\hline Director/a, ejectivo/a o alta habilidad & $\begin{array}{c}0.117 \\
(0.096)\end{array}$ & $* *$ \\
\hline Contrato a tiempo completo & $\begin{array}{c}0.134 \\
(0.091)\end{array}$ & * \\
\hline Contrato a tiempo parcial & $\begin{array}{c}0.059 \\
(0.103)\end{array}$ & \\
\hline Amo/a de casa & $\begin{array}{c}0.092 \\
(0.097)\end{array}$ & \\
\hline Estudiante & $\begin{array}{c}0.067 \\
(0.115)\end{array}$ & \\
\hline Sin trabajo & $\begin{array}{c}0.119 \\
(0.096)\end{array}$ & ** \\
\hline
\end{tabular}

$\mathrm{F}=20.746^{* * *}$; R2 Ajustada=0.093; error típico=0.754; n=2492.

Nota: Para cada variable se muestra el coeficiente de regresión estandarizado y el error típico entre paréntesis. ${ }^{* * *} \mathrm{p}<0.01,{ }^{* *} \mathrm{p}<0.05,{ }^{*} \mathrm{p}<0.10$

Fuente: elaboración propia a partir de Oficina de Gabinete, Gobierno de Japón, 2013. 
ingresos familiares o personas con contrato a tiempo completo que aseguren la estabilidad económica), más habilidades cívicas (cargos ejecutivos o de dirección con mayores redes sociales) y más tiempo libre (se puede interpretar que las personas sin trabajo son mayoritariamente jubilados y tienen más tiempo libre, al contrario que los que tienen más niños, lo que implicaría una mayor falta de tiempo libre), tienden a expresar más interés por los temas políticos.

La edad aparece como la variable explicativa con un mayor peso. La posible explicación puede que esté relacionada con el tiempo libre, ya que el ser mayor, probablemente en situación de jubilación, implicaría una mayor disposición de tiempo libre para dedicarse a leer, informarse, hablar, pensar e interesarse en la política. Por último, se confirma un mayor interés por la política en los hombres que en las mujeres, en línea con la literatura previa (Verba et al., 1997; Burns et al., 2001; Tiefenbach y Holdgrün, 2015; Takeda, 2010).

En resumen, estos resultados permiten confirmar la H0 (influencia positiva de la participación en actividades cívicas sobre el interés en la política), si bien la magnitud de su efecto es pequeña en comparación con la de otras variables. Podríamos afirmar que la participación cívica tiene menos influencia sobre el aumento del interés por la política en el caso de Japón; sin embargo, al no contar con resultados para otros países utilizando la misma evidencia, no podemos afirmar una excepcionalidad japonesa en este sentido. Al mismo tiempo, resulta necesario señalar el posible problema de lo endógeno de efectos entre variables y la dificultad para discernir entre los efectos recíprocos de la causalidad entre las distintas variables consideradas. Esto es, la participación cívica en asociaciones puede ser tanto causa como efecto del interés por la política. Aun así, la evidencia apunta a favor de la hipótesis de Vinken et al. (2010) respecto de la falta de interés en los asuntos políticos entre los japoneses en general.

En segundo lugar, analizamos si el factor de voluntariedad o no voluntariedad sobre la participación en actividades cívicas afecta al grado de interés por la política. A la especificación de la ecuación previa se añaden la variable dicotómica de voluntariedad (valor 1 = genuina voluntariedad). Introducimos tres modelos diferentes para intentar dar respuesta a las hipótesis 1 y $2(\mathrm{H} 1$ y H2). El modelo 1 es el modelo básico de regresión lineal que considera a las variables de participación cívica y voluntariedad de la participación como variables independientes. Como se muestra en la tabla 2 , la participación en actividades voluntarias sigue mostrándose positiva y significativa. Además, el resto de variables sociodemográficas y económicas producen efectos muy similares sobre el interés por la política a los obtenidos como resultado en la tabla 1 . En cambio, el efecto tanto de la voluntariedad como de la no voluntariedad no parece ser relevante, lo que apunta a que no hay suficiente evidencia como para aceptar H1. Podría convenirse que es más bien el efecto de la participación en asociaciones, al margen de que podamos desentrañar la genuina voluntariedad de participación en las mismas, el que ha de considerarse relevante para entender el impacto sobre el interés por la política. Esto es algo que han constatado, por otra parte, la mayor parte de los estudios culturalistas referidos al papel socializador y potenciador de las virtudes cívicas de los mecanismos internos del compromiso asociativo. 
TABLA 2.

REGRESIÓN LINEAL DE INTERÉS EN POLÍTICA (VS. VOLUNTARIEDAD Y NO VOLUNTARIEDAD)

\begin{tabular}{|c|c|c|c|}
\hline (Variables independientes) & Modelo 1 & Modelo 2 & Modelo 3 \\
\hline $\begin{array}{l}\text { Participación en } \\
\text { actividades cívicas }\end{array}$ & $\begin{array}{c}0.128^{* *} \\
(0.009)\end{array}$ & $\begin{array}{r}0.078 \\
(0.034)\end{array}$ & $\begin{array}{l}0.153^{* *} \\
(0.031)\end{array}$ \\
\hline $\begin{array}{l}\text { Voluntariedad para la } \\
\text { participación } \\
\text { (1: voluntario; } \\
0: \text { no voluntario) }\end{array}$ & $\begin{array}{l}-0.067 \\
(0.059)\end{array}$ & $\begin{array}{r}0.024 \\
(0.108)\end{array}$ & $\begin{array}{l}-0.114 \\
(0.022)\end{array}$ \\
\hline Sexo & $\begin{array}{r}0.142^{* * *} \\
(0.058)\end{array}$ & & \\
\hline Edad & $\begin{array}{r}0.356^{* * *} \\
(0.002) \\
\end{array}$ & $\begin{array}{r}0.358^{* * *} \\
(0.011) \\
\end{array}$ & $\begin{array}{r}0.389 \\
(0.005) \\
\end{array}$ \\
\hline Ingresos de la familia & $\begin{array}{c}0.133^{* *} \\
(0.013) \\
\end{array}$ & $\begin{array}{c}0.168^{* *} \\
(0.028) \\
\end{array}$ & $\begin{array}{r}0.128 \\
(0.036) \\
\end{array}$ \\
\hline Casado/a & $\begin{array}{l}-0.072 \\
(0.156) \\
\end{array}$ & $\begin{array}{r}-0.120 \\
(0.184) \\
\end{array}$ & $\begin{array}{l}-0.081 \\
(0.182)\end{array}$ \\
\hline Número de hijos/as & $\begin{array}{l}-0.218 \\
(0.043)^{* * *}\end{array}$ & $\begin{array}{l}-0.298 \\
(0.059)^{* * *}\end{array}$ & $\begin{array}{l}-0.178 \\
(0.054)^{* *}\end{array}$ \\
\hline Hijos/as menores de 6 & $\begin{array}{r}0.034 \\
(0.098) \\
\end{array}$ & $\begin{array}{c}0.113^{*} \\
(0.156)\end{array}$ & $\begin{array}{r}0.012 \\
(0.134) \\
\end{array}$ \\
\hline $\begin{array}{l}\text { Director/a, ejecutivo/a o } \\
\text { alta habilidad }\end{array}$ & $\begin{array}{r}0.133 \\
(0.278)\end{array}$ & $\begin{array}{c}0.356^{* *} \\
(0.291)\end{array}$ & $\begin{array}{l}-0.035 \\
(0.348)\end{array}$ \\
\hline $\begin{array}{l}\text { Contrato a tiempo } \\
\text { completo }\end{array}$ & $\begin{array}{l}0.293^{* *} \\
(0.172)\end{array}$ & $\begin{array}{r}0.498^{* * *} \\
(0.256)\end{array}$ & $\begin{array}{l}0.114^{*} \\
(0.219)\end{array}$ \\
\hline $\begin{array}{l}\text { Contrato a tiempo } \\
\text { parcial }\end{array}$ & $\begin{array}{r}0.009 \\
(0.216) \\
\end{array}$ & $\begin{array}{r}0.032 \\
(0.379) \\
\end{array}$ & $\begin{array}{r}-0.047 \\
(0.287) \\
\end{array}$ \\
\hline Amo/a de casa & $\begin{array}{c}0.156^{*} \\
(0.234) \\
\end{array}$ & $\begin{array}{l}0.228^{* *} \\
(0.356) \\
\end{array}$ & $\begin{array}{r}0.018 \\
(0.271) \\
\end{array}$ \\
\hline Estudiante & $\begin{array}{l}0.174^{* *} \\
(0.253) \\
\end{array}$ & $\begin{array}{l}0.222^{* *} \\
(0.384) \\
\end{array}$ & $\begin{array}{r}0.101 \\
(0.049) \\
\end{array}$ \\
\hline Sin trabajo & $\begin{array}{r}0.123 \\
(0.260)\end{array}$ & $\begin{array}{r}0.421^{* * *} \\
(0.293)\end{array}$ & $\begin{array}{c}0.178^{*} \\
(0.387)\end{array}$ \\
\hline $\mathrm{F}$ & $7.943^{* * *}$ & $4.634^{* * *}$ & $3.937^{* * *}$ \\
\hline $\mathrm{R}^{2}$ Ajustada & 0,183 & 0,169 & 0,145 \\
\hline Error típico & 0,694 & 0,647 & 0,683 \\
\hline $\mathrm{N}$ & 597 & 307 & 290 \\
\hline
\end{tabular}

Nota: para cada variable se muestra el coeficiente de regresión estandarizado y el error típico entre paréntesis ${ }^{* * *} \mathrm{p}<0.01$, ${ }^{* *} \mathrm{p}<0.05,{ }^{*} \mathrm{p}<0.10$.

Fuente: elaboración propia a partir de los datos de la Oficina de Gabinete, Gobierno de Japón, 2013. 
Finalmente, observamos las posibles diferencias por razón de sexo en el efecto de la voluntariedad sobre el interés por la política. Lo hacemos siguiendo la técnica utilizada por Burns et al. (2001) de subdividir la estimación para hombres y para mujeres dada la diferencia en los procesos de socialización que a veces influyen a los hombres y no a las mujeres y viceversa (Burns et al., 2001: 39). Los modelos 2 y 3 de la tabla 2 muestran esta estimación ${ }^{4}$. El modelo 2 cuenta solo las muestras de hombres y el 3 de mujeres. En el modelo 3, la variable de no voluntariedad en la participación cívica resulta significativa en un sentido negativo. Es decir, la no voluntariedad en la participación cívica para las mujeres, así como su menor interés en los asuntos políticos, pese a que no es la variable más discriminante. Respecto al resto de variables, para el caso del modelo 2 las que parecen nuevamente significativas coinciden mayormente con las del modelo 1; esto es, concretamente las variables sociodemográficas económicas. Este resultado apoya la importancia de los recursos sociodemográficos y económicos que se argumenta en el modelo de voluntarismo cívico.

Por otro lado, el modelo 3, al margen de la consideración anterior, apenas presenta variables explicativas significativas que sean útiles para revelar los factores influyentes del interés en política, por lo que tampoco coinciden con algunos de los hallazgos sobre el voluntarismo cívico. Por lo tanto, los estudios señalados previamente sobre la indiferencia política en general de las mujeres japonesas (Masuyama y Yamada, 2004; Oyama, 2002; Takeda, 2010) serían más compatibles con el resultado del modelo 3. También se podría interpretar que la apatía política de las mujeres en Japón es mucho mayor que el factor de voluntariedad o de no voluntariedad respecto del interés hacia cuestiones sociopolíticas. En conclusión, el factor de voluntariedad o no voluntariedad muestra menos relevancia sobre el nivel de interés por la política; precisamente, se observa solo este efecto en el caso de las mujeres. Por ello podría confirmarse la segunda parte de $\mathrm{H} 2$.

\section{CONCLUSIONES}

En este estudio se han examinado algunas de las hipótesis clásicas de las teorías de la sociedad civil, el capital social y el voluntarismo cívico que sostienen la idea del poder de las asociaciones cívicas para aumentar el interés por la política y promocionar la participación política. Hemos intentado cuestionar la afirmación manifestada en muchas de esas teorías acerca de la idea de que las asociaciones cívicas voluntarias están siempre unidas por las voluntades de sus miembros. Existe un número importante de miembros de estas asociaciones que mantienen su participación al estar presionados

4. Como puede observarse, se ha replicado la estimación en la tabla 1 separadamente para hombres y mujeres, obteniéndose que el efecto de la participación en asociaciones es muy parecido para los dos géneros, tal y como se constata igualmente en la tabla 5 del anexo. 
socialmente, culturalmente o por otros motivos. De este modo, hemos propuesto dos hipótesis generales para comprobar estas teorías utilizando el caso de Japón. Asumimos como premisa que se trata de un espacio donde se observan asociaciones cívicas organizadas no esencialmente de forma voluntaria, sino donde la involucración de los individuos podría explicarse más bien por la costumbre, la estructura social o la conveniencia política.

La primera conclusión del presente trabajo es que si bien la participación en las diversas actividades cívicas fomenta el interés en la política, en el caso de la sociedad japonesa no resulta especialmente llamativa la relación entre ambas variables. Otras variables sociodemográficas económicas muestran una relación empírica de mayor magnitud. En segundo lugar, en cuanto a la voluntariedad o la no voluntariedad de participación en las actividades cívicas, no se ha podido constatar una incidencia clara de las mismas en el caso de Japón. No obstante, cuando se analizan los grupos de hombres y mujeres por separado, si bien la voluntariedad en ambos grupos no muestra una influencia relevante, la no voluntariedad muestra una cierta mayor incidencia sobre el nivel de interés por la política en el caso de las mujeres, con lo que confirma que la no voluntariedad en la participación en actividades cívicas afecta negativamente al nivel de interés en la política. Otros factores relacioandos con el interés por la política coinciden aproximadamente con los hallazgos de las tesis del voluntarismo cívico, salvo el caso de las mujeres. En conclusión, si bien de forma provisoria, podemos señalar que la voluntariedad (o no voluntariedad) sí es relevante a la hora de entender las causas del interés en la política.

En sociedades como la japonesa, que incluyen muchas asociaciones cívicas voluntarias, pero donde el grado de influencia de la voluntariedad y la no voluntariedad parece tener importancia -causado, muy probablemente, por sus costumbres, cultura o presión social-, el sistema sociopolítico sí que cuenta para su funcionamiento con esas asociaciones y actividades cívicas. Sin embargo, la ausencia de voluntariedad para participar en esas asociaciones no parece fomentar la capacidad de los ciudadanos para relacionar los problemas en sus comunidades cercanas con los más lejanos, para así tomar conciencia de las cuestiones nacionales o internacionales, y finalmente interesarse en asuntos sociales y políticos en general.

La apatía política o la participación obligada pueden poner en riesgo la democracia de un país. La importancia del poder de las asociaciones cívicas voluntarias como escuelas de democracia debe enfrentarse al hecho de que los miembros de las mismas no deben ser obligados o presionados para pertenecer a ellas. Igualmente, si la indiferencia política es observada solo entre una población limitada -en nuestro concretamente en las mujeres-, hay que continuar investigando las causas y razones de las diferencias de género, con el objetivo de que la participación cívica sea auténticamente equilibrada y representativa de toda la población. Hay que admitir, por tanto, que los resultados de esta investigación no son suficientemente sustanciales para apoyar en su totalidad la hipótesis de la influencia de la falta de voluntariedad, además de las diferencias de género. Quedamos a la espera de obtener más recursos para avanzar en los análisis y desarrollar estas y futuras hipótesis. 


\section{AGRADECIMIENTOS}

Los autores muestran su agradecimiento a la Oficina de Gabinete del Gobierno de Japón por ofrecerles y facilitarles el uso de los datos de la ENPEV 2012.

\section{Referencias}

Almond, Gabriel y Sidney Verba. 1963. The civic culture: political attitudes and democracy in five nations. Princeton: Princeton University Press. Disponible en: https:// doi.org/10.1515/9781400874569.

Ayala, Louis. 2000. «Trained for democracy: The differing effects of voluntary and involuntary organizations on political participation», Political Research Quarterly, 53 (1): 99-115. Disponible en: https://doi.org/10.1177/106591290005300105.

Barber, Benjamin R. 1984. Strong democracy: Participatory politics for a new age. Berkeley: University of California Press.

Bell, Daniel. 2006. Beyond liberal democracy: Political thinking for an east Asian context. Princeton, N. J.: Princeton University Press. Disponible en: https://doi. org/10.1515/9781400827466.

Bell, Daniel. 2016. "Communitarianism», en Edward N. Zalta (ed.), The Stanford encyclopedia of philosophy (summer 2016 edition). Disponible en: http://plato.stanford.edu/archives/sum2016/entries/communitarianism/.

Bellah, Robert N., Richard Madsen, William M. Sullivan, Ann Swidler and Steven M. Tipton. 2007. Habits of the Heart: Individualism and Commitment in American Life. Berkeley: University of California Press.

Beng-Huat, Chau. 1999. «Asian-values' discourse and the resurrection of the social», East Asia Cultures Critique, 7 (2): 573-592. Disponible en: https://doi. org/10.1215/10679847-7-2-573.

Boix, Carles y Daniel N. Posner. 1998. «Social capital: Explaining its origins and effects on government performance», British Journal of Political Science, 24 (4): 686-693. Disponible en: https://doi.org/10.1017/S0007123498000313.

Burns, Nancy, Kay L. Schlozman y Sidney Verba. 2001. The private roots of public action: Gender, equality and political participation. Cambridge, Massachusetts; London: Harvard University Press.

Chan, Joseph. 1997. "An alternative view», Journal of Democracy, 8 (2): 35-48. Disponible en: https://doi.org/10.1353/jod.1997.0018.

Cohen, Joshua y Joel Rogers. 1992. «Secondary associations and democratic governance», Politics and Society, 20 (4): 393-472. Disponible en: https://doi.org/10.11 77/0032329292020004003.

Dekker, Paul y Loek Halman. 2003. The values of volunteering: cross-cultural perspectives. New York: Kluwer Academic/Plenum Publishers. Disponible en: https://doi. org/10.1007/978-1-4615-0145-9. 
Delli Carpini, Michael X. y Scott Keeter. 1992. "The gender gap in political knowledge», The Public Perspective, 3 (5): 23-26.

Ellis, Susan y Katherine Campbell. 2005. By the people: A history of Americans as volunteers. Philadelphia: Energize.

Ellison, Christopher y Darren Sherkat. 1995. "The 'semi-involuntary institution' revisited: Regional variations in church participation among black Americans", Social Forces, 73 (4): 1415-1437. Disponible en: https://doi.org/10.2307/2580453.

Eztioni, Amitai. 1993. The spirit of community: rights, responsibilities, and the communitarian agenda. New York: Crown Publishers.

Etzioni, Amitai, Andrew Volert y Elanit Rothschild. 1994. The communitarian reader: beyond the essentials. Lanham: Rowman and Littlefield Publishers.

Fraile, Marta y Raúl Gómez. 2017. «Bridging the enduring gender gap in political interest in Europe: The relevance of promoting gender equality», European Journal of Political Research. Disponible en: htpps://doi.org/10.1111/1475-6765.12200

Gelb, Joyce. 2003. Gender policies in Japan and the United States. New York, Hampshire:PalgraveMacmillan.Disponibleen:https://doi.org/10.1057/9781403976789.

Haddad, Mary. 2007. Politics and volunteering in Japan: A global perspective. Cambridge, etc.: Cambridge University Press. Disponible en: https://doi.org/10.1017/ CBO9780511510120.

Haddad, Mary. 2010. "The state-in-society approach to the study of democratization with examples from Japan», Democratization, 17 (5): 997-1023. Disponible en: https://doi.org/10.1080/13510347.2010.501182.

Holdgrün, Phoebe y Barbara Holthus. 2014. Gender and political participation in post3/11 Japan. Deutsches Institut Für Japanstudien working paper 14/3. Disponible en: http://www.dijtokyo.org/publications/WP1403_Holdgruen_Holthus.pdf.

Inglehart, Ronald y Pippa Norris. 2003. Rising tide: Gender equality and cultural change around the world. Cambridge: Cambridge University Press. Disponible en: ttps://doi.org/10.1017/CBO9780511550362.

Kingston, Jeff. 2004. Japan's quiet transformation: Social change and civil society in the 21st century. London: Routledge.

LeBlanc, Robin. 1999. Bicycle citizens: The political world of Japanese housewife. Berkeley: University of California Press.

Maan, Xandra. 2011. Social capital and civic voluntarism: A socio-political explanation of political participation. Bachelor thesis, University of Twnte. Disponible en: http://essay.utwente.nl/62739/1/Bscverslag_XandraMaan_s0191094.pdf.

Masuyama, Mikitaka y Masahiro Yamada. 2008. «Seiji sanka no zyendā gyappu (diferencia de género sobre la participación política)», en Mikitaka Masuyama (ed.), Keiryō seiji bunsei nyūmon (Introducción al análisis cuantitativa de la ciencia política). Tokio: Tōkyō daigaku syuppan kai.

Morales Diez de Ulzurrun, Laura. 2002. "Associational membership and social capital in comparative perspective: A note on the problems of measurement», Politics and Society, 3 (3): 497-523. Disponible en: https://doi.org/10.1177/0032329202 030003005 . 
Newton, Kenneth. 1999. «Social capital and democracy in modern Europe», en Jan W van Deth, Marco Maraffi, Ken Newton y Paul F. Whiteley (eds.), Social capital and European democracy. London: Routledge.

Ōyama, Nao. 2002. «Seiji teki taido to kōdō ni miru zyendā (comportamiento político y el género)», Tōkai daigaku kiyō bungaku bu (Boletín del Departamento de Literatura de la Universidad de Tökai), 78: 167-183.

Paxton, Pamela. 2002. «Social capital and democracy: An interdependent relationship». American Sociological Review, 67 (2): 254-277. Disponible en: https://doi. org/10.2307/3088895.

Pateman, Carole. 1970. Participation and democratic theory. Cambridge: Cambridge University Press. Disponible en: https://doi.org/10.1017/CBO9780511720444.

Pekkanen, Robert. 2004. "Japan: social capital without advocacy», en M. Alagappa (ed.), Civil society and political change in Asia: expanding and contracting democratic space. Stanford, California: Stanford University Press.

Pekkanen, Robert. 2006. Japan's dual civil society: Members without advocates. Stanford, California: Stanford University Press.

Pérez-Fuentes, Pilar. 1990. «Condicionamientos de la participación política de las mujeres», en Judith Astelarra (comp.), Participación politica de las mujeres. Madrid: Centro de Investigaciones Sociológicas.

Persell, Caroline H., Adam Green y Liena Gurevich. 2001. «Civil society, economic distress, and social tolerance», Sociological Forum, 16 (2): 203-230. Disponible en: https://doi.org/10.1023/A:1011048600902.

Pidgeon, Walter P. (1998). The universal benefits of volunteering: A practical workbook for nonprofit organizations, volunteers, and corporations. New York: Wiley.

Putnam, Robert. 2000. Bowling alone: The collapse and revival of American community. New York: Simon and Schuster. Disponible en: https:/doi.org/10.1145/ 358916.361990.

Putnam, Robert, Robert Leonardi y Raffaella Nanetti. 1993. Making democracy work: Civic traditions in modern Italy. Princeton, New Jersey: Princeton University Press.

Pye, Lucian W. y Mary W. Pye. 1985. Asian power and politics: The cultural dimensions of authority. Cambridge, Massachusetts: Belknap Press.

Rawls, John. 1971. A theory of justice. Cambridge, Massachusetts: Harvard University Press.

Rosenblum, Nancy L. 1998. Membership and morals: The personal uses of pluralism in America. Princeton, Chichester: Princeton University Press.

Sandel, Michael J. 1998. Liberalism and the limits of justice. Cambridge: Cambridge University Press. Disponible en: https://doi.org/10.1017/CBO9780511810152.

Schlozman, Key L., Nancy Burns y Sidney Verba. 1994. "Gender and the pathways to participation: The role of resources», The Journal of Politics, 56 (4): 963-990. Disponible en: https://doi.org/10.2307/2132069.

Schlozman, Key L., Sidney Verba y Henry E. Brady. 2012. The unheavenly chorus: Unequal political voice and the broken promise of American democracy. Princeton, Oxford: Princeton University Press. Disponible en: https://doi.org/10.1515/9781400841912. 
Schwartz, Frank y Susan Pharr (eds.). 2003. The state of civil society in Japan. Cambridge: Cambridge University Press.

Takeda, Yuka. 2010. «Seiji sanka ni okeru gyendā gyappu (Diferencias de género sobre la participación política)», JGSS Research Series, 7: 323-335.

Tanaka, Minoru. 2000. Sekai no zyūmin soshiki: azia to öbei no kokusai hikaku (Asociaciones de vecinos en el mundo: comparaciones internacionales entre paises asiáticos y occidentales). Tokio: Zichitai kenkyū sha.

Tiefenbach, Tim y Phoebe Holdgrün. 2015. «Happiness through participation in neighborhood associations in Japan? The impact of loneliness and voluntariness», Voluntas, 26: 69-97. Disponible en: https://doi.org/10.1007/s11266-014-9520-8.

Tiefenbach, Tim y Florian Kohlbacher. 2013. Happiness and life satisfaction in Japan by gender and age. Deutsches Institut Für Japanstudien working paper, 13/2. Disponible en: http://www.dijtokyo.org/publications//WP1302_Tiefenbach_Kohlbacher.pdf.

Tocqueville, Alexis de. 2003. Democracy in America. London: Penguin Books.

Torigoe, Hiroyuki. 1994. Chiiki zichikai no kenkyü (Estudio sobre asociaciones de vecinos locales). Tokio: Mineruva shobō.

Tu, Wei-ming (ed.). 1996. Confucian traditions in East Asia modernity: Moral education and economic culture in Japan and the four mini-dragons. Cambridge, Massachusetts: Harvard University Press.

Van Deth, Jan W. (2006) «Democracy and involvement. The benevolent aspects of social participation», en Mariano Torcal y José Ramón Montero (eds.), Political disafecction in contemporary democracies. Social capital, institutions and politics. London: Routledge.

Vázquez García, Rafael. 2010. Compromiso cívico y democracia: los efectos democráticos del asociacionismo sociopolítico en España. Sevilla: Fundación Pública Andaluza Centro de Estudios Andaluces.

Verba, Sidney, Kay L. Schlozman y Henry E. Brady. 1995. Voice and equality. Cambridge, Massachusetts: Harvard University Press.

Verba, Sidney, Nancy Burns y Kay L. Schlozman. 1997. «Knowing and caring about politics: gender and political engagement», The Journal of Politics, 59 (4): 10511072. Disponible en: https://doi.org/10.2307/2998592.

Verba, Sidney, Kay L. Schlozman y Henry E. Brady. 2002. Voice and equality: Civic voluntarism in American politics. Cambridge, Massachusetts: Harvard University Press.

Verge, Tania. y Raúl Tormos. 2012. "The persistence of gender differences in political interest», Revista Española de Investigaciones Sociológicas, 138: 89-108. Disponible en: https://doi.org/10.5477/cis/reis.138.89.

Vinken, Henk, Yuko Nishimura, Bruce L.J. White y Masayuki Deguchi (eds.). 2010. Civic engagement in contemporary Japan: Established and emerging repertories. New York, Dordrecht, Heidelberg, London: Springer.

Warren, Mark. 2001. Democracy and association. Princeton, New Jersey: Princeton University Press. 
Wilson, Victoria. 2007. "The associational roots of the political participation gender gap», paper delivered at the 2007 Midwest Political Science Association Meetings, Chicago.

Wollebaek, Dag y Per Selle. 2002. "Does participation in voluntary associations contribute to social capital? The impact of intensity, scope, and type», Nonprofit and Voluntary Sector Quarterly, 31 (1): 32-61. Disponible en: https://doi. org/10.1177/0899764002311002.

Wollebaek, Dag y Per Selle. 2003. «The importance of passive membership for social capital formation", en Marc Hooghe y Dietlind Stolle (eds.), Generating social capital: Civil society and institutions in comparative perspective. New York: Palgrave Macmillan. Disponible en: https://doi.org/10.1057/9781403979544_4.

Yamazaki, Takeo. 1999. Chiiki no zidai no chōnai kai, zichi kai, comyunithi (Asociaciones de vecinos en la era de locales). Tokio: Zichitai kenkyū sha.

\section{ANEXO 1}

Ficha técnica sobre National Survey of Lifestyle Preferences 2012 (Encuesta Nacional sobre las Preferencias de Estilos Vida, KoKumin SEIKATSu senKō do Chōsa)

1. Objetivo de la encuesta

Junto con la conciencia pública relacionada con la felicidad y el nuevo público, esta encuesta pretende comprender los cambios a largo plazo de los ciudadanos sobre las ideas acerca de la vida en general y del área de bienestar.

2. Encuestados

- Población: hombres y mujeres de edades entre más de quince y menos de ochenta años que residen en todo Japón.

- Tamaño de la muestra: 4000 personas.

- Método de elección: procedimiento aleatorio en dos etapas y estratificado.

3. Período de investigación

21 de marzo hasta 30 de marzo de 2012 (10 días).

4. Método de investigación

Visitas individuales por los examinadores.

5. Agencia delegada de encuesta

Survey Research Center.

6. Número válido de respuestas (\%)

2802 personas $(70,1 \%)$.

Fuente: Oficina de Gabinete, Gobierno de Japón, 2013. Traducción propia. 


\section{ANEXO 2}

\section{Preguntas de los Cuestionarios utilizados}

Q9. ¿Usted está participando en las siguientes actividades voluntarias, actividades sin ánimo de lucro o actividades cívicas por la forma de participación directa, utiliza los servicios o mediante donación? Si participa, marque todos los números aplicados en las áreas de actividad. Si no participa en ninguno, marque «14. ninguno» (las respuestas corresponden a las participaciones, usos de servicios o donaciones en 2013. Además, si participa en algunas actividades, no limite los actos sin compensaciones, sino también las actividades de las que haya recibido coste real o compensaciones).

TABLA 3.

PARTICIPACIÓN, USO DE SERVICIOS O DONACIONES EN 2013

\begin{tabular}{lccc}
\hline & $\begin{array}{c}(\mathbf{a}) \\
\text { Participación } \\
\text { directa }\end{array}$ & $\begin{array}{c}(\mathbf{i}) \\
\text { Utilizar } \\
\text { servicios }\end{array}$ & $\begin{array}{c}(\mathbf{u}) \\
\text { Mediante } \\
\text { Donación }\end{array}$ \\
\hline 1. Educación & $(3,4)$ & $(1,7)$ & $(1,3)$ \\
\hline 2. Cuidar a los niños/as & $(5,4)$ & $(3,2)$ & $(1,1)$ \\
\hline 3. Desarrollo de comunidad & $(7,4)$ & $(1,3)$ & $(1,9)$ \\
\hline 4. Prevención de crímenes y desastres & $(7,1)$ & $(1,1)$ & $(1,1)$ \\
\hline 5. Cuidar a los mayores y bienestar & $(4,8)$ & $(3,4)$ & $(3,0)$ \\
\hline 6. Promoción de salud & $(4,6)$ & $(3,6)$ & $(0,4)$ \\
\hline 7. Promoción de actividades académicas, deportes, Cul- & $(6,3)$ & $(3,4)$ & $(1,4)$ \\
\hline tura y arte & $(3,3)$ & $(0,5)$ & $(1,5)$ \\
\hline $\begin{array}{l}\text { 8. } \text { Medioambiente y medidas contra calentamiento } \\
\text { global }\end{array}$ & $(3,6)$ & $(0,4)$ & $(32,3)$ \\
\hline $\begin{array}{l}\text { 9. Rescate y recuperación de desastres y cooperación } \\
\text { internacional }\end{array}$ & $(2,4)$ & $(0,2)$ & $(25,6)$ \\
\hline $\begin{array}{l}\text { trucción del gran terremoto de Japón Oriental } \\
\text { 10. Promoción de las TIC }\end{array}$ & $(0,5)$ & $(1,0)$ & $(0,1)$ \\
\hline 11. Desarrollo de habilidades de trabajo & $(0,8)$ & $(0,7)$ & $(0,3)$ \\
\hline $\begin{array}{l}\text { 12. Promoción del interés de los consumidores } \\
\text { 13. Otras }\end{array}$ & $(0,3)$ & $(0,5)$ & $(0,3)$ \\
\hline \begin{tabular}{l} 
14. Ninguno \\
\hline
\end{tabular} & $(1,7)$ & $(0,5)$ & $(2,0)$ \\
\hline
\end{tabular}

(a) $\mathrm{n}=2783$, (i) $\mathrm{n}=2756$, (u) $\mathrm{n}=2765$.

[Solo los que han marcado cualquier área de la pregunta Q9 (a) (los que han marcado cualquiera de 1-14), contesten a la pregunta Q10]. 
Q10, 1). ¿Cuáles son las razones de participación? (los que están participando en varias actividades de varias áreas, marquen todas las razones aplicables).

$(n=676)$

1. Porque siento una motivación en mi vida $(29,4)$.

2. Porque quiero contribuir a la sociedad $(42,6)$.

3. Porque disfruto participando en ellas $(40,8)$.

4. Porque puedo hacer amistades $(41,1)$.

5. Porque tienen que ayudarse mutuamente $(36,8)$.

6. Porque quiero aumentar ingresos $(3,1)$.

7. Porque es obligatorio participar puesto que los cargos turnan $(23,1)$.

8. Otras (concretar) $(4,4)$.

Q19. En su caso, ¿en qué medida se aplica la siguiente pregunta sobre opinión o actitud?

(Pregunta U) Yo tengo interés en los problemas sociales y los asuntos políticos más que otras personas. $(\mathrm{n}=2790)$

(1) Totalmente de acuerdo $(10,3)$.

(2) Más o menos de acuerdo $(44,4)$.

(3) Más o menos no estar de acuerdo $(36,4)$.

(4) Totalmente no estar de acuerdo $(8,9)$.

Nota: Los porcentajes de respuesta entre paréntesis.

Fuente: Oficina de Gabinete, Gobierno de Japón, 2013. Traducción propia.

\section{ANEXO 3}

TABLA 4.

RESUMEN DE LAS MUESTRAS DE ENPEV 2013

\begin{tabular}{lccccc}
\hline \multicolumn{1}{c}{ Variables } & N & M & DT & Min & Max \\
\hline (Variable dependiente) & & & & & \\
\hline Interés en política & 2790 & 2,56 & 0,794 & 1 & 4 \\
\hline (Variables independientes) & & & & & \\
\hline Suma de participación en las actividades cívicas & 2740 & 1,28 & 2,111 & 0 & 16 \\
\hline Voluntariedad de la participación (dummy) & 676 & 0,8 & 0,4 & 0 & 1 \\
\hline No voluntariedad de la participación (dummy) & 676 & 0,55 & 0,498 & 0 & 1 \\
\hline (Variables de Control) & & & & & \\
\hline Hombre (dummy) & 2802 & 0,48 & 0,5 & 0 & 1 \\
\hline Edad & 2802 & 49,3 & 17,251 & 15 & 79 \\
\hline $10 s$ (dummy) & 2802 & 0,05 & 0,225 & 0 & 1 \\
\hline & & & & & $\ldots . \ldots$
\end{tabular}




\begin{tabular}{lccccc}
\hline \multicolumn{1}{c}{ Variables } & N & M & DT & Min & Max \\
\hline 20s (dummy) & 2802 & 0,1 & 0,294 & 0 & 1 \\
\hline 30s (dummy) & 2802 & 0,16 & 0,365 & 0 & 1 \\
\hline 40s (dummy) & 2802 & 0,19 & 0,391 & 0 & 1 \\
\hline 50s (dummy) & 2802 & 0,16 & 0,37 & 0 & 1 \\
\hline 60s (dummy) & 2802 & 0,2 & 0,399 & 0 & 1 \\
\hline 70 s (dummy) & 2802 & 0,349 & 0,349 & 0 & 1 \\
\hline 80 (dummy) & 2802 & 0 & 0 & 0 & 1 \\
\hline Ingresos de la familia & 2566 & 3,14 & 1,683 & 1 & 8 \\
\hline Relaciones familiares & & & & & \\
\hline casado/a (dummy) & 2973 & 0,24 & 0,43 & 0 & 1 \\
\hline número de hijos/as & 2769 & 1,42 & 1,154 & 0 & 4 \\
\hline si tiene uno o más hijos/as menores de 6 (dummy) & 2802 & 0,8 & 0,279 & 0 & 1 \\
\hline Información profesional (dummy) & & & & & \\
\hline $\begin{array}{l}\text { Con cargo de director, ejectivo o alta habilidad } \\
\text { como profesor, médico, técnico, escritor, etc. }\end{array}$ & 2802 & 0,16 & 0,365 & 0 & 1 \\
\hline contrato a tiempo completo & 2802 & 0,34 & 0,474 & 0 & 1 \\
\hline contrato a tiempo parcial & 2802 & 0,9 & 0,284 & 0 & 1 \\
\hline amo/a de casa & 2802 & 0,16 & 0,366 & 0 & 1 \\
\hline estudiante & 2802 & 0,06 & 0,243 & 0 & 1 \\
\hline sin trabajo & 2802 & 0,16 & 0,363 & 0 & 1 \\
\hline
\end{tabular}

Fuente: elaboración propia a partir de los datos de la Oficina de Gabinete, Gobierno de Japón, 2013.

\section{ANEXO 4}

\section{TABLA 5.}

REgRESIÓN LINEAL DE INTERÉS EN POLÍTICA (VS. PARTICIPACIÓN EN ACTIVIDADES CÍVICAS) (HOMBRES Y MUJERES POR SEPARADO)

\begin{tabular}{|c|c|c|c|c|}
\hline (Variables independientes) & Hombres & & Mujeres & \\
\hline Participación en actividades cívicas & $\begin{array}{c}0.076 \\
(0.006)\end{array}$ & $* * *$ & $\begin{array}{c}0.072 \\
(0.005)\end{array}$ & *** \\
\hline \multicolumn{5}{|l|}{ (Variables de control) } \\
\hline Edad & $\begin{array}{c}0.250 \\
(0.002)\end{array}$ & $* * *$ & $\begin{array}{c}0.332 \\
(0.002)\end{array}$ & $* * *$ \\
\hline
\end{tabular}




\begin{tabular}{|c|c|c|c|c|}
\hline (Variables de control) & Hombres & & Mujeres & \\
\hline Ingresos de la familia & $\begin{array}{c}0.086 \\
(0.015) \\
\end{array}$ & $* * *$ & $\begin{array}{c}0.123 \\
(0.013) \\
\end{array}$ & $* * *$ \\
\hline Casado/a & $\begin{array}{c}0.000 \\
(0.081)\end{array}$ & & $\begin{array}{l}-0.010 \\
(0.080)\end{array}$ & \\
\hline Número de hijos/as & $\begin{array}{l}-0.041 \\
(0.028 \\
\end{array}$ & & $\begin{array}{l}-0.114 \\
(0.025)\end{array}$ & $* * *$ \\
\hline Hijos/as menores de 6 & $\begin{array}{c}0.032 \\
(0.092)\end{array}$ & & $\begin{array}{c}0.053 \\
(0.078)\end{array}$ & $* *$ \\
\hline $\begin{array}{l}\text { Director/a, ejectivo/a o alta } \\
\text { habilidad }\end{array}$ & $\begin{array}{c}0.280 \\
(0.134)\end{array}$ & $* * *$ & $\begin{array}{l}-0.038 \\
(0.140)\end{array}$ & \\
\hline Contrato a tiempo completo & $\begin{array}{c}0.289 \\
(0.128) \\
\end{array}$ & $* * *$ & $\begin{array}{l}-0.042 \\
(0.129) \\
\end{array}$ & \\
\hline Contrato a tiempo parcial & $\begin{array}{l}0.033 \\
(0.220)\end{array}$ & & $\begin{array}{l}-0.057 \\
(0.135)\end{array}$ & \\
\hline Amo/a de casa & $\begin{array}{c}0.084 \\
(0.171)\end{array}$ & * & $\begin{array}{l}-0.053 \\
(0.131)\end{array}$ & \\
\hline Estudiante & $\begin{array}{c}0.114 \\
(0.170) \\
\end{array}$ & $* * *$ & $\begin{array}{l}-0.025 \\
(0.156)\end{array}$ & \\
\hline Sin trabajo & $\begin{array}{c}0.272 \\
(0.137) \\
\end{array}$ & $* * *$ & $\begin{array}{l}-0.061 \\
(0.136)\end{array}$ & \\
\hline $\mathrm{F}$ & 8602 & $* * *$ & 9504 & $* * *$ \\
\hline $\mathrm{R}^{2}$ Ajustada & 0,07 & & 0,074 & \\
\hline Error típico & 0,786 & & 0,719 & \\
\hline $\mathrm{n}$ & 1212 & & 1280 & \\
\hline
\end{tabular}

Nota: Para cada variable se muestra el coeficiente de regresión estandarizado y el error típico entre paréntesis. ${ }^{* * *} \mathrm{p}<0.01,{ }^{* *} \mathrm{p}<0.05,{ }^{*} \mathrm{p}<0.10$.

Fuente: elaboración propia a partir de los datos de la Oficina de Gabinete, Gobierno de Japón, 2013.

Presentado para evaluación: 12 de septiembre de 2016.

Aceptado para publicación: 21 de mayo de 2017.

\section{KYOTO ITO-MORALES}

kito@correo.ugr.es

Estudiante de doctorado vinculada al Departamento de Ciencia Política y de la Administración de la Universidad de Granada, donde acaba de finalizar su tesis doctoral sobre la «Naturaleza del asociacionismo y el capital social en la democracia japonesa». Licenciada en Ciencias Políticas y Relaciones Internacionales por la International 
Christian University de Tokyo (Japón). MA en Theory and Practice of Human Rights por el Human Rights Centre de la Universidad de Essex (Reino Unido). Investigadora visitante en la Universidad Pontificia Católica de Chile.

RAFAEL VÁZQUEZ-GARCÍA

rvazquez@ugr.es

Profesor contratado doctor en el Departamento de Ciencia Política y de la Administración de la Universidad de Granada. Premio Nacional de Finalización de Estudios en Ciencia Política en 2001. Investigador visitante en varias universidades europeas (MZES, LSE entre otras). Está especialmente interesado en el estudio de la sociedad civil (desde el asociacionismo cívico a la desobediencia civil), así como en otras cuestiones de teoría política, como la teoría política verde y el feminismo, los derechos animales y el antiespecismo, la teoría crítica y la teoría política no occidental. Es autor de libros, capítulos de libro y artículos en las áreas referidas. 